\title{
CELDAS SOLARES SENSIBILIZADAS POR COLORANTE BASADAS EN TiO2 MODIFICADO CON CuO
}

\author{
Vanessa C. Martínez ${ }^{\mathrm{a}}$, Alcides López ${ }^{\mathrm{a}}$, Mónica M. Gómez ${ }^{* a}$
}

\begin{abstract}
RESUMEN
Se prepararon celdas solares sensibilizadas con colorante empleando como electrodo de trabajo $\mathrm{TiO}_{2}$ modificado con $\mathrm{CuO}\left(\mathrm{TiO}_{2}-\mathrm{CuO}\right)$. En la preparación del recubrimiento modificado se utilizó un polvo comercial de $\mathrm{TiO}_{2}$ (P25-Degussa) y pequeñas cantidades de $\mathrm{CuO}$ en polvo. El recubrimiento se depositó por el método del doctor Blade, sobre un sustrato conductor de $\mathrm{SnO}_{2}: \mathrm{F}$, en el cual se depositó previamente, por la técnica de rociado pirolítico, una película delgada de $\mathrm{TiO}_{2}$, como capa de bloqueo. Para la sensibilización del recubrimiento se usó el complejo de rutenio, cis-bis (isotiocianato) bis (2,2'bipiridil-4,4'dicarboxilato) rutenio (II) bistetrabutil amonio, también conocido como N719. El polvo de $\mathrm{CuO}$ fue caracterizado estructuralmente por difracción de rayos X y su morfología fue estudiada por microscopía electrónica de barrido. Los grupos funcionales de los compuestos usados, $\mathrm{TiO}_{2}$ y $\mathrm{CuO}$ fueron estudiados por espectroscopía infrarroja. Mediante voltametría cíclica se mostraron los ciclos de oxidación/reducción del $\mathrm{Cu}^{+1} / \mathrm{Cu}^{+2}$. Por medio de las medidas de eficiencia de conversión fotón incidente a electrón generado, y las medidas de eficiencia solar, se obtuvo que las películas $\mathrm{TiO}_{2}-\mathrm{CuO}$ modificadas con el $0,33 \%$ de $\mathrm{CuO}$ mostraron valores de $57 \%$ y 2,0\%, respectivamente, que son mayores a los que se prepararon solo con $\mathrm{TiO}_{2}$, que fueron $52 \%$ y $1,7 \%$, respectivamente.
\end{abstract}

Palabras clave: Celdas solares sensibilizadas, $\mathrm{TiO}_{2}, \mathrm{CuO}$, capa de bloqueo.

\section{DYE SENSITIZED SOLAR CELLS BASED ON TiO, MODIFIED WITH CuO}

\begin{abstract}
Some dye-sensitized solar cells were prepared using $\mathrm{TiO}_{2}$ modified with $\mathrm{CuO}\left(\mathrm{TiO}_{2}-\mathrm{CuO}\right)$ like a working electrode. P25 commercial powder and small amounts of $\mathrm{CuO}$ powder were used in preparing the modified coating. The coating was deposited following doctor Blade method, on a conductive substrate $\mathrm{SnO}_{2}: \mathrm{F}$, which was previously deposited on a $\mathrm{TiO}_{2}$ blocking layer by spray pyrolysis technique. For sensitization of the coating, a ruthenium complex, cis-bis (isothiocyanate) bis (2,2'bipiridil-4,4'dicarboxilato) ruthenium (II) ammonium
\end{abstract}

\footnotetext{
${ }^{a}$ Facultad de Ciencias, Universidad Nacional de Ingeniería, Av. Túpac Amaru 210, Lima 25, Perú. mgomez@uni.edu.pe
} 
bistetrabutil, also known as N719, was used. The $\mathrm{CuO}$ powder was structurally characterized by X-ray diffraction and its morphology was studied by scanning electron microscopy. The functional groups of $\mathrm{TiO}_{2}$ and $\mathrm{CuO}$ were studied by infrared spectroscopy. The oxidation and reduction cycles of $\mathrm{Cu}$ were shown by cyclic voltammetry. Through incident photon to current conversion efficiency measures, and solar efficiency measures, it was found that the modified films $\mathrm{TiO}_{2}-\mathrm{CuO}$ with $0,33 \% \mathrm{CuO}$ displayed values of 57 and $2 \%$, respectively, which were higher than those prepared from $\mathrm{TiO}_{2}$, which were $52 \%$ and $1,7 \%$, respectively.

Key words: Dye solar cells, $\mathrm{TiO}_{2}, \mathrm{CuO}$, blocking layer.

\section{INTRODUCCIÓN}

La preocupación por el medio ambiente ha venido incrementándose considerablemente en estos últimos años, con la búsqueda de fuentes de energías renovables 1 y la implementación de nuevas tecnologías que las aprovechen de manera eficiente.

Una de estas nuevas tecnologías son las celdas solares sensibilizadas por colorante, también llamadas DSC (de los términos en inglés Dye-sensitized solar cells), que se vienen desarrollando desde la década de los 90 's $^{2}$ y que están constituidas por un electrodo de trabajo mesoporoso. Además, a diferencia de las celdas convencionales, son de fácil fabricación y de bajo costo.

El principio de funcionamiento de estas celdas está basado en procesos de transferencia interfacial de electrones. Al incidir sobre el fotoelectrodo la radiación $(h v)$ es absorbida por el sensibilizador o colorante $(S)$, anclado sobre el semiconductor, que pasa a su estado excitado $\left(S^{*}\right)^{3}$ :

$$
S+h v \rightarrow S^{*}
$$

A partir del estado excitado del colorante $\left(S^{*}\right)$ se produce la inyección de electrones hasta los estados disponibles en la banda de conducción del $\mathrm{TiO}_{2}$, y a su vez los electrones se difunden a través de las nanopartículas hasta el sustrato conductor:

$$
S^{*} \rightarrow S^{+}+e^{-} \text {(inyectado en el semiconductor) }
$$

El circuito se cierra externamente en el contraelectrodo, donde los electrones reducen el $I_{3}$ - a I- (que forma parte electrolito), y que será la especie que repondrá electrones al nivel desocupado en el colorante, cerrando el ciclo de corriente:

$$
\begin{array}{lll}
I_{3}^{-}+2 e^{-}(P t) & \rightarrow & 3 I^{-} \\
S^{+}+3 / 2 I^{-} & \rightarrow & S+1 / 2 I_{3}^{-}
\end{array}
$$

Durante este ciclo se producen procesos no deseados que suponen una pérdida de la eficiencia de la celda solar. Los electrones inyectados en la banda de conducción del $\mathrm{TiO}_{2}$ pueden transferirse al colorante oxidado (ecuación 5) o a los iones $I_{3}^{-}$, que se encuentran cercanos a la superficie del semiconductor (ecuación 6):3 


$$
\begin{aligned}
& S^{+}+e^{-}\left(\mathrm{TiO}_{2}\right) \rightarrow S \\
& \mathrm{I}_{3^{-}}+2 e^{-}\left(\mathrm{TiO}_{2}\right) \rightarrow 3 I^{-}
\end{aligned}
$$

Para obtener buenas eficiencias es necesario que los procesos de inyección (ecuación 2) y regeneración (ecuación 4) estén cinéticamente favorecidos con respecto a los procesos de recombinación (ecuaciones 5 y 6 ).

Otro proceso clave en el mecanismo de funcionamiento de las DSC es la absorción de luz por el colorante. Cuando el colorante absorbe la luz del sol, un electrón es excitado desde el nivel del orbital molecular ocupado de mayor energía (HOMO de los términos en inglés higest occupied molecular orbital) al nivel del orbital molecular desocupado de menor energía (LUMO de los términos en inglés lowest unoccupied molecular orbital). Un punto importante a considerar es la posición relativa entre estos orbitales del colorante y las bandas de energía del semiconductor, para asegurar la transferencia electrónica. La figura 1 muestra el esquema de niveles de energía propuestos para una $\mathrm{DSC}^{4}$.

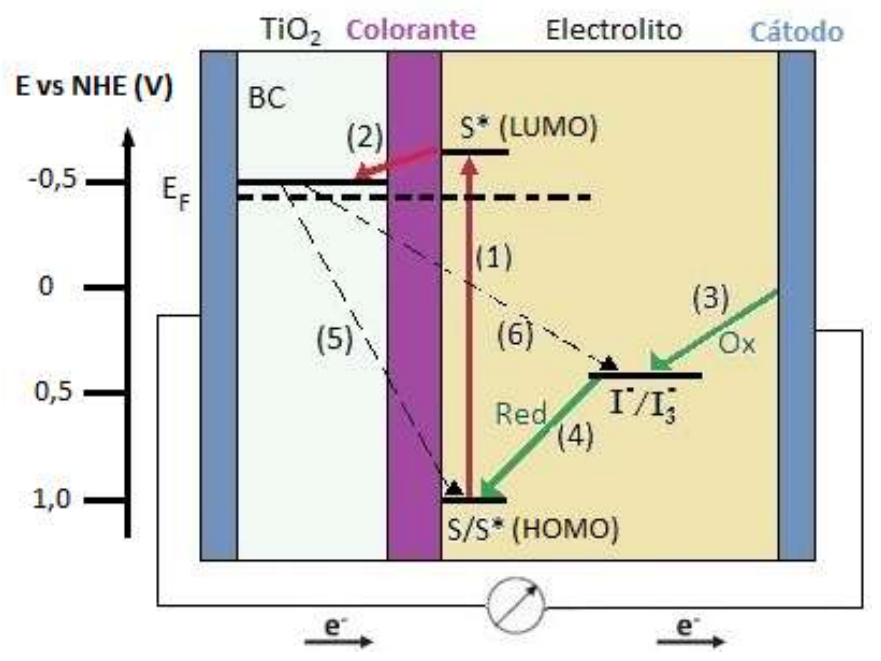

Figura 1. Esquema del funcionamiento de una DSC. Los procesos que tienen lugar son: excitación (1), inyección (2), reducción del electrolito (3), regeneración del colorante (4). Durante el ciclo pueden producirse pérdidas debido a transferencia de electrones representadas por 5 y 6 (Adaptado de ${ }^{4}$ ).

Además de los componentes mencionados, se han realizado estudios en los que otros óxidos como $\mathrm{NiO}^{5}, \mathrm{MgO}, \mathrm{SnO}_{2}$ se han agregado al $\mathrm{TiO}_{2}$ mesoporoso para modificarlo y mejorar la eficiencia de las celdas. En el presente trabajo se usó como modificante el $\mathrm{CuO}$, lo que constituyó un recubrimiento de $\mathrm{TiO}_{2}$ con partículas de $\mathrm{CuO}$ homogéneamente distribuidas en la película, que en adelante se denominará $\mathrm{TiO}_{2}-\mathrm{CuO}$. 


\section{PARTE EXPERIMENTAL}

A continuación, se muestra la parte experimental del trabajo y las caracterizaciones realizadas, a los componentes del electrodo de trabajo y a la celda solar.

\section{Depósito de la capa de bloqueo de $\mathrm{TiO}_{2}$}

Esta capa de bloqueo6 de $\mathrm{TiO}_{2}$ se depositó sobre el sustrato de vidrio conductor $\left(\mathrm{SnO}_{2}: \mathrm{F}\right)$ por la técnica de rociado pirolítico, en la configuración final se encuentra entre la película mesoporosa de $\mathrm{TiO}_{2}$ y el sustrato conductor. La solución precursora a rociar fue isopropóxido de titanio diluido en alcohol isopropílico, en porcentaje de 20 a $80 \%$, y manteniendo el sustrato a $350{ }^{\circ} \mathrm{C}$ de temperatura.

Las reacciones que se postulan serían las que evolucionan en el camino que recorre la nube desde la solución hasta el sustrato ${ }^{7}$.

$\begin{array}{ll}\text { Hidrólisis } & \mathrm{Ti}(\mathrm{OR})_{4}+4 \mathrm{H}_{2} \mathrm{O} \rightarrow 2 \mathrm{Ti}(\mathrm{OH})_{4}+4 \mathrm{ROH} \\ \text { Condensación } & \mathrm{Ti}(\mathrm{OH})_{4} \rightarrow \mathrm{TiO}_{2} \mathrm{xH}_{2} \mathrm{O}+(2-x) \mathrm{H}_{2} \mathrm{O} \\ \text { Deshidratación } & \mathrm{TiO}_{2} x \mathrm{H}_{2} \mathrm{O} \rightarrow \mathrm{TiO}_{2}+\mathrm{H}_{2} \mathrm{O}\end{array}$

En la figura 2 se muestra el esquema del equipo de rociado pirolítico usado en el laboratorio para depositar las capas de bloqueo. En este proceso se realizó un estudio del número de recorridos del sustrato con los que las celdas de $\mathrm{TiO}_{2}-\mathrm{CuO}$ alcanzaron la mejor eficiencia solar.

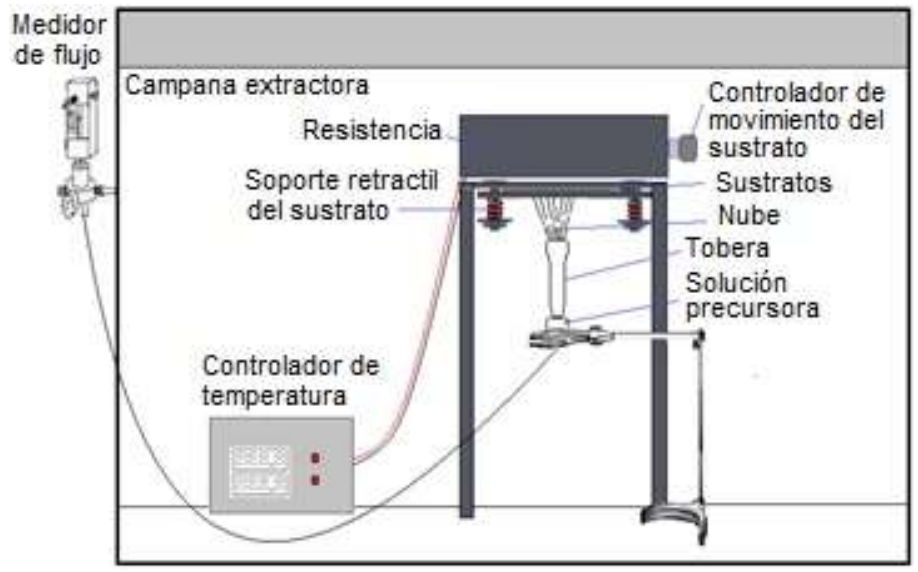

Figura 2. Esquema del equipo de rociado pirolítico. 


\section{Síntesis de partículas de $\mathrm{CuO}$}

El óxido de cobre, empleado en el recubrimiento $\mathrm{TiO}_{2}-\mathrm{CuO}$, se sintetizó preparando una solución hidro-alcohólica de sulfato de cobre $(5 \mathrm{mM})$, que se colocó dentro de un baño ultrasónico (Cole-Parmer - $42 \mathrm{kHz}, 130 \mathrm{~W}$ )8, por $1,5 \mathrm{~h}$ a $60{ }^{\circ} \mathrm{C}$. Luego se llevó a un pH 9 por medio de gotas de hidróxido de amonio al $25 \%$, que es el punto en el que empieza la precipitación del $\mathrm{CuO}$. El polvo de $\mathrm{CuO}$ obtenido se lavó agregando agua destilada, se centrifugó y secó a $80{ }^{\circ} \mathrm{C}$ durante 2 horas.

\section{Elaboración del electrodo de trabajo modificado ( $\left.\mathrm{TiO}_{2}-\mathrm{CuO}\right)$}

Para la preparación del recubrimiento, se diluyó $0,12 \mathrm{~g}$ de etil celulosa y en $1 \mathrm{ml}$ de etanol, se agitó durante unos minutos, hasta volverse una solución densa; aparte se pesaron $0,8 \mathrm{~g}$ de $\alpha$-terpineol y se le agregó $0,5 \mathrm{ml}$ de etanol, se agitó manualmente. Luego se agregó la cantidad de $\mathrm{CuO}$ deseada (de 0 a $1,67 \%$ p/p, ver tabla 1), respecto al polvo de $\mathrm{TiO}_{2}$ (Degussa P25). Se agitó un par de minutos y se agregó la solución de etil celulosa con etanol. Para homogenizar la pasta, se aplicó un proceso intercalado de agitación magnética y ultrasonido durante algunos minutos, en la figura 3 se describe gráficamente esta preparación. Luego, la pasta modificada $\mathrm{TiO}_{2}-\mathrm{CuO}$ obtenida fue depositada sobre el vidrio conductor por el método del doctor Blade ${ }^{9}$. Estos recubrimientos, luego de secarse por unas horas, fueron sometidos a tratamiento térmico, con una rampa de $4{ }^{\circ} \mathrm{C} / \mathrm{min}$ hasta $500{ }^{\circ} \mathrm{C}$. Finalmente, estos recubrimientos fueron sensibilizados, es decir, fueron sumergidos en una solución alcohólica del colorante, de concentración $0,5 \mathrm{mM}$, durante 15 horas en oscuridad. Finalmente, se procedió a sellar la celda, que consistió en colocar una capa de Surlyn, entre el electrodo de trabajo y el contraelectrodo, derritiéndola hasta que selle. En el contraelectrodo, previamente, se hizo 2 orificios, por uno de ellos se inyectó el electrolito y se colocó una capa de Surlyn y, posteriormente, un vidrio para sellar dichos orificios, como se muestra en la figura 3.

Tabla 1. Valores de \% peso del $\mathrm{CuO}$ respecto al $\mathrm{TiO}_{2}$, usados en el trabajo.

\begin{tabular}{c}
\hline$\% \mathbf{p} / \mathbf{p ~ C u O} / \mathrm{TiO}_{2}$ \\
\hline 0,00 \\
0,17 \\
0,33 \\
0,67 \\
1,00 \\
1,33 \\
1,67 \\
\hline
\end{tabular}




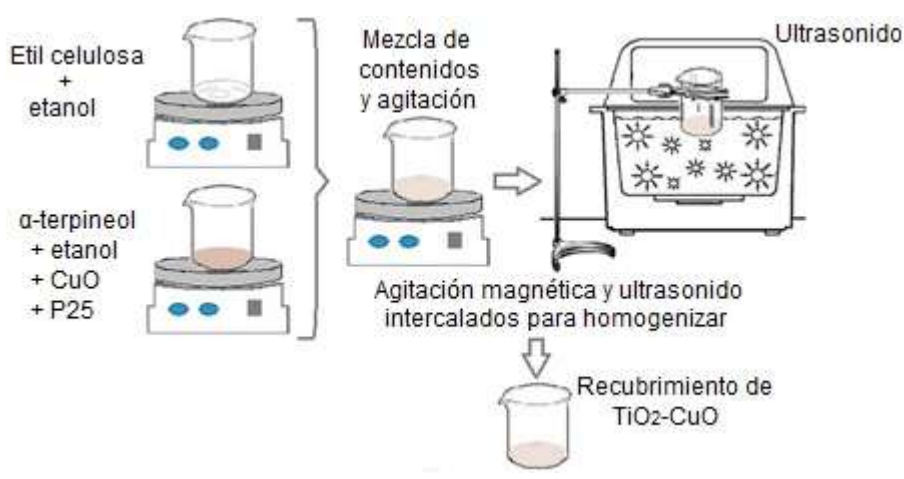

Figura 3. Esquema de la preparación del electrodo de trabajo constituido por recubrimientos de $\mathrm{TiO}_{2}-\mathrm{CuO}$.

\section{RESULTADOS Y DISCUSIÓN}

A continuación, se presentan los resultados de las caracterizaciones realizadas.

\section{Difracción de Rayos X}

La caracterización por DRX de las muestras de $\mathrm{TiO}_{2}$ y $\mathrm{CuO}$ fueron analizadas por el difractómetro RIGAKU Miniflex II Desktop X Ray, con ánodo de $\mathrm{Cu}$, operado con radiación $\operatorname{CuK} \alpha(\lambda=0,1540562 \mathrm{~nm})$, en un montaje experimental convencional $\theta-2 \theta$.

La figura 4 muestra el patrón de difracción de una capa de bloqueo de $\mathrm{TiO} 2$ obtenida por rociado pirolítico. Los picos de difracción corresponden a las reflexiones (101), (004), (200), (211), (105) y (204) de la fase anatasa del $\mathrm{TiO}_{2}$ para los ángulos 25, 38, 48, 54, 55 y 63, respectivamente. Empleando el programa TOPAS10 se obtuvo que el tamaño promedio de los cristales para la fase anatasa fue de $60 \mathrm{~nm}$.

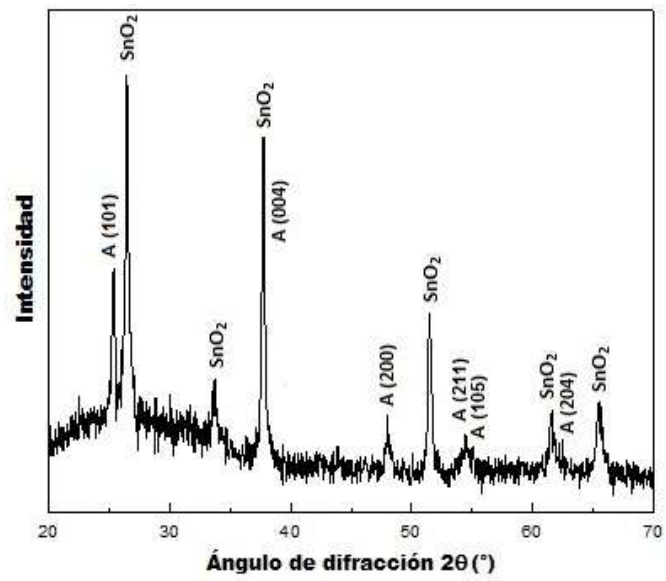

Figura 4. Difractograma de rayos $\mathrm{X}$ para una capa de bloqueo de $\mathrm{TiO}_{2}$ depositada por rociado pirolítico sobre sustrato de vidrio recubierto de $\mathrm{SnO}_{2}: \mathrm{F}$. 
La figura 5 muestra los difractogramas de rayos $\mathrm{X}$ de una serie de 3 muestras en polvo de $\mathrm{CuO}$, sometidas a tratamiento térmico de 80,250 y $500^{\circ} \mathrm{C}$. Con el programa TOPAS, el tamaño de los cristales resultó 9,9 y $10 \mathrm{~nm}$ para las temperaturas de 80,250 y $500^{\circ} \mathrm{C}$, respectivamente. Se observa que este rango de temperatura no afecta el tamaño de los dominios cristalinos.

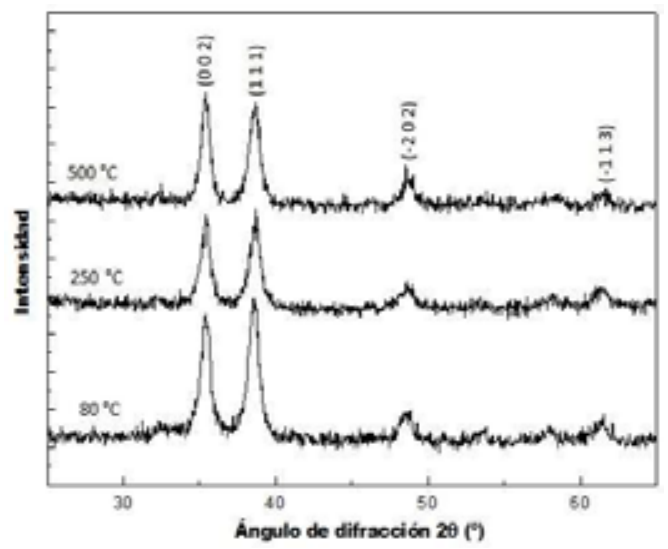

Figura 5. Difractogramas de rayos $\mathrm{X}$ de partículas de $\mathrm{CuO}$, con tratamientos térmicos a diferentes temperaturas.

\section{Microscopía electrónica de barrido}

El microscopio electrónico de barrido, usado en este trabajo, fue el Vega3 TESCAN, operado a $20 \mathrm{kV}$. La figura 6 corresponde a las micrografías electrónicas de barrido de partículas de $\mathrm{CuO}$, con tratamientos térmicos de 80 y $500{ }^{\circ} \mathrm{C}$, ambos polvos muestran conglomerados de formas ovoides, laminares y esféricas, con tamaños que varían desde 50 hasta $500 \mathrm{~nm}$. Se evidencia que en este rango de temperatura los conglomerados cristalinos no modifican su morfología, de manera análoga que, para el caso de los dominios cristalinos, observados por DRX.
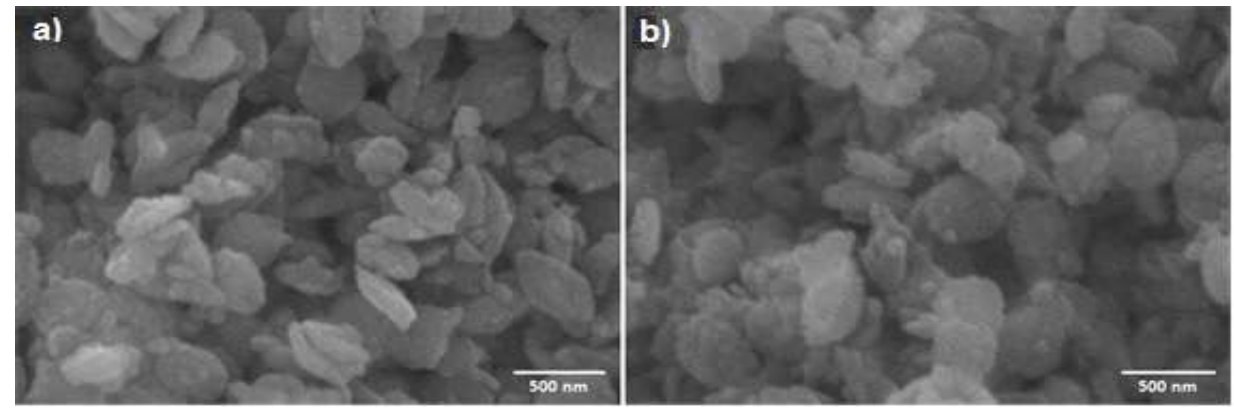

Figura 6. Micrografía electrónica de barrido de partículas de $\mathrm{CuO}$. a) $80^{\circ} \mathrm{C}$, b) $500{ }^{\circ} \mathrm{C}$. 
La figura 7 muestra micrografías de una película de $\mathrm{TiO}_{2}-\mathrm{CuO}(0,33 \% \mathrm{p} / \mathrm{p})$ depositada sobre sustrato de vidrio pre-cubierto con $\mathrm{SnO}_{2}: \mathrm{F}$. En estas micrografías (de distintas amplificaciones) se observa una distribución uniforme de los conglomerados cristalinos, los cuales presentan un tamaño $\sim 100 \mathrm{~nm}$, además se observan escasas cavidades superficiales. De estas micrografías no se reconoce la presencia de $\mathrm{CuO}$ en el $\mathrm{TiO}_{2}$

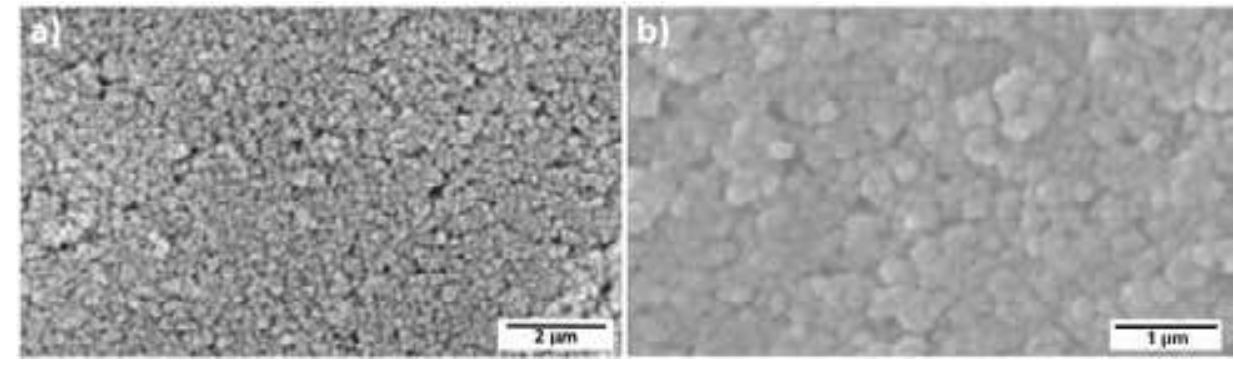

Figura 7. Micrografía electrónica de barrido de una película de $\mathrm{TiO}_{2}-\mathrm{CuO}$. a) 13 kX y (b) 27 kX de magnificación.

La figura 8 corresponde a la micrografía electrónica de barrido de la sección transversal de un electrodo de trabajo, el cual constó de un sustrato de vidrio pre-cubierto con $\mathrm{SnO}_{2}: \mathrm{F}$, sobre el cual se depositó una capa de bloqueo de $\mathrm{TiO}_{2}$ por rociado pirolítico y sobre esta se depositó una película mesoporosa de $\mathrm{TiO}_{2}$, por el método del doctor Blade. En la figura se indican las posiciones de estos materiales y se estima que el grosor de la película mesoporosa es de $20 \mu \mathrm{m}$, aproximadamente, mientras que la película delgada o capa de bloqueo de $\mathrm{TiO}_{2}$ no se logró resolver por esta técnica.

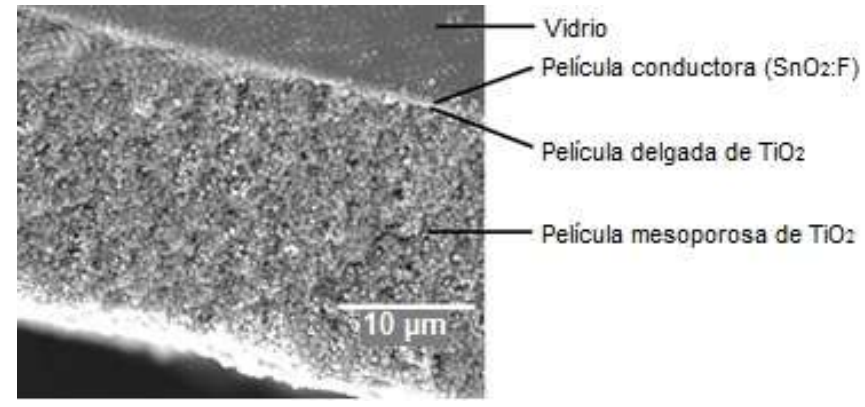

Figura 8. Micrografía electrónica de barrido de una película de $\mathrm{TiO}_{2}-\mathrm{CuO}$.

\section{Espectroscopía infrarroja transformada de Fourier}

Estas medidas se realizaron usando el espectrómetro infrarrojo IR Prestige 21 Shimadzu en el modo transmisión. Para analizar las muestras de $\mathrm{TiO}_{2}$ y $\mathrm{CuO}(0,0005 \mathrm{~g})$ se prepararon pastillas con $\mathrm{KBr}(0,06 \mathrm{~g})$. La figura 9 muestra los espectros FTIR, para las muestras en polvo de $\mathrm{TiO}_{2}, \mathrm{TiO}_{2}-\mathrm{CuO}$ y $\mathrm{CuO}$. Los espectros de $\mathrm{TiO}_{2}$ y $\mathrm{TiO}_{2}-\mathrm{CuO}$ muestran un pico amplio en $580 \mathrm{~cm}-1$ debido al $\mathrm{TiO}_{2}$ asignado al modo stretching del enlace O-Ti- $\mathrm{O}^{11}$. Los espectros 
de $\mathrm{TiO}_{2}-\mathrm{CuO}$ son bastante parecidos al de $\mathrm{TiO}_{2}$, no se observan picos adicionales debido a la adición de $\mathrm{CuO}$. En el espectro de $\mathrm{CuO}$ se observan picos a 426, 546 y $597 \mathrm{~cm}^{-1}$ que se asignan a los modos stretching característicos del enlace $\mathrm{Cu}-\mathrm{O}^{12}$.

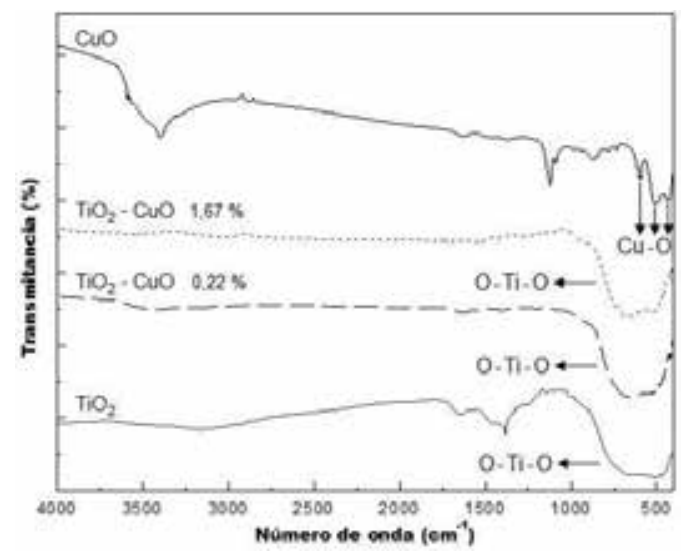

Figura 9. Espectros FTIR de nanopartículas de $\mathrm{TiO}_{2}, \mathrm{TiO}_{2}-\mathrm{CuO}$ y $\mathrm{CuO}$.

\section{Curva característica corriente - voltaje (I-V)}

El montaje de medición experimental constó de una lámpara de $250 \mathrm{~W}$, que brindó una irradiancia de $1000 \mathrm{~W} / \mathrm{m}^{2}$ sobre la celda evaluada de área $0,25 \mathrm{~cm}^{2}$, un banco óptico, un filtro IR, y un sistema de adquisición de datos voltaje-corriente. Todo el sistema se ubicó dentro de una cámara oscura ${ }^{5}$. Para verificar si la capa de bloqueo disminuyó procesos de transferencia de electrones que representen pérdidas, se fabricaron películas con diferente número de recorridos de rociado pirolítico, para obtener diferentes espesores, donde de cada recorrido se espera tener un espesor aproximado de $10 \mathrm{~nm}^{6}$. Luego se evaluaron sus curvas I-V. La figura 10 muestra los resultados obtenidos.
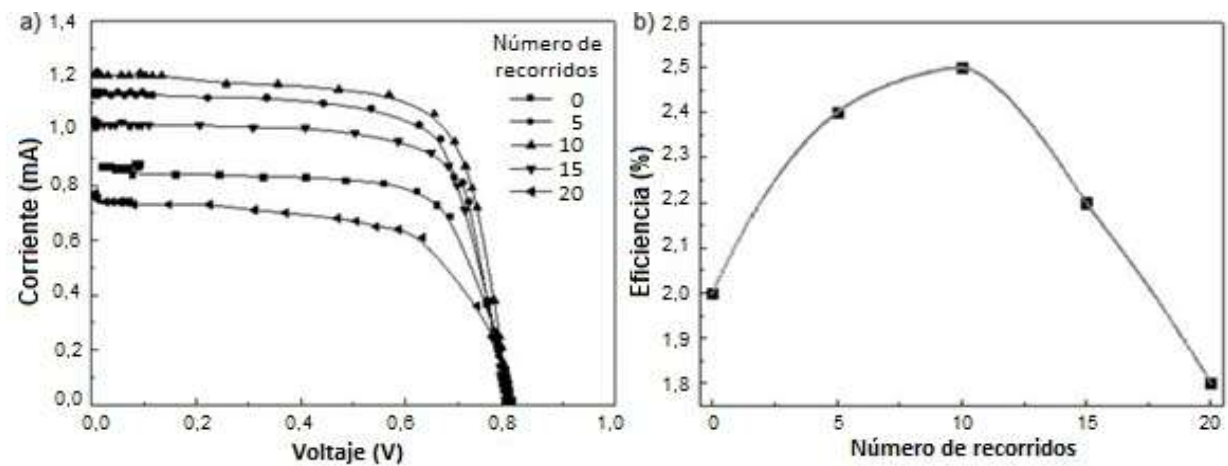

Figura 10. a) Curvas $\mathrm{I}-\mathrm{V}$ de celdas de $\mathrm{TiO}_{2}$ mesoporoso con capas de bloqueo de diferente número de recorridos del sistema de rociado pirolítico, b) eficiencia solar de la celda vs número de recorridos del sistema de rociado pirolítico. 
Se observa que la mejor eficiencia corresponde a la capa de bloqueo que tiene 10 recorridos del sistema de rociado pirolítico, lo que correspondería a un espesor aproximado de $100 \mathrm{~nm}$. Después de determinar el valor óptimo para la capa de bloqueo se procedió a elaborar las películas $\mathrm{TiO}_{2}-\mathrm{CuO}$, tal como se describió en la figura 3, variando la cantidad de $\mathrm{CuO}$, de acuerdo a la tabla 1. La figura 11 muestra las curvas I-V obtenidas, donde la mejor eficiencia se observa para la celda construida con el recubrimiento $\mathrm{TiO}_{2}-\mathrm{CuO}$, al $0,33 \% \mathrm{p} / \mathrm{p}$ de $\mathrm{CuO}$.

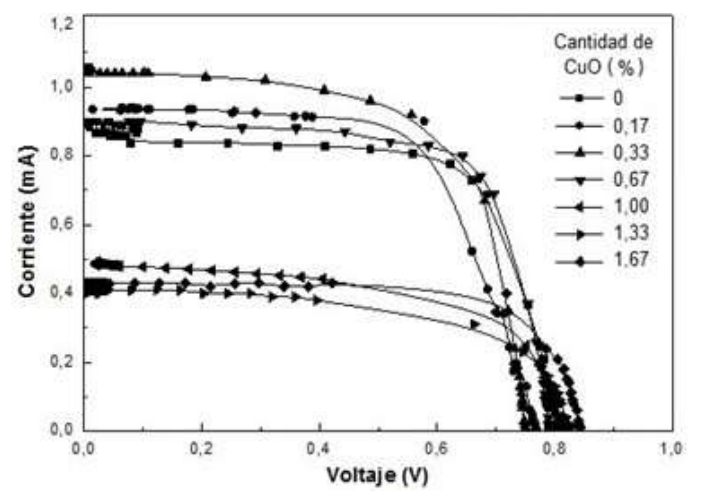

Figura 11. Curvas I - V obtenidas con diferentes cantidades de \% p/p de $\mathrm{CuO}$.

Se observó que para las celdas, cuyos recubrimientos contuvieron $0,17,0,33$ y $0,67 \% \mathrm{p} / \mathrm{p}$ de $\mathrm{CuO}$, la corriente aumentó con respecto a la celda de solo $\mathrm{TiO}_{2}$, especialmente la que contuvo $0,33 \%$ de $\mathrm{CuO}$; mientras que los valores de Voc variaron entre 0,77 y 0,79 V, lo que originó un aumento de eficiencias. Por otro lado, para las celdas cuyos recubrimientos contuvieron $1,00,1,33$ y $1,67 \%$ p/p de $\mathrm{CuO}$ se observó una disminución abrupta de la corriente, disminuyendo su eficiencia con respecto a la celda de solo $\mathrm{TiO}_{2}$. Sin embargo, los valores de Voc presentaron un ligero aumento $(0,81$ a $0,84 \mathrm{~V})$. La tabla 2 muestra en detalle los parámetros de corriente de corto circuito (Jsc), voltaje de circuito abierto (Voc), factor de forma (FF) y eficiencia $(\eta)$ para cada una de estas celdas, y estos se grafican en la figura 12 en función de la concentración del $\mathrm{CuO}$.

Tabla 2. Performance de las celdas sensibilizadas basadas en electrodos de $\mathrm{TiO} 2-\mathrm{CuO}$.

\begin{tabular}{ccccc}
\hline Muestra \% p/p CuO & Jsc $\left(\mathbf{m A} / \mathbf{c m}^{2}\right)$ & Voc (V) & FF (\%) & $\eta(\mathbf{\%})$ \\
\hline $\mathrm{TiO}_{2}(0 \%)$ & 3,52 & 0,80 & 0,69 & 1,7 \\
$\mathrm{TiO}_{2}-\mathrm{CuO}(0,17 \%)$ & 3,75 & 0,77 & 0,72 & 1,8 \\
$\mathrm{TiO}_{2}-\mathrm{CuO}(0,33 \%)$ & 4,20 & 0,75 & 0,65 & $\mathbf{2 , 0}$ \\
$\mathrm{TiO}_{2}-\mathrm{CuO}(0,67 \%)$ & 3,60 & 0,79 & 0,72 & 1,6 \\
$\mathrm{TiO}_{2}-\mathrm{CuO}(1,00 \%)$ & 1,67 & 0,81 & 0,72 & 1,0 \\
$\mathrm{TiO}_{2}-\mathrm{CuO}(1,33 \%)$ & 1,64 & 0,82 & 0,59 & 0,8 \\
$\mathrm{TiO}_{2}-\mathrm{CuO}(1,67 \%)$ & 1,68 & 0,84 & 0,81 & 0,8 \\
\hline
\end{tabular}



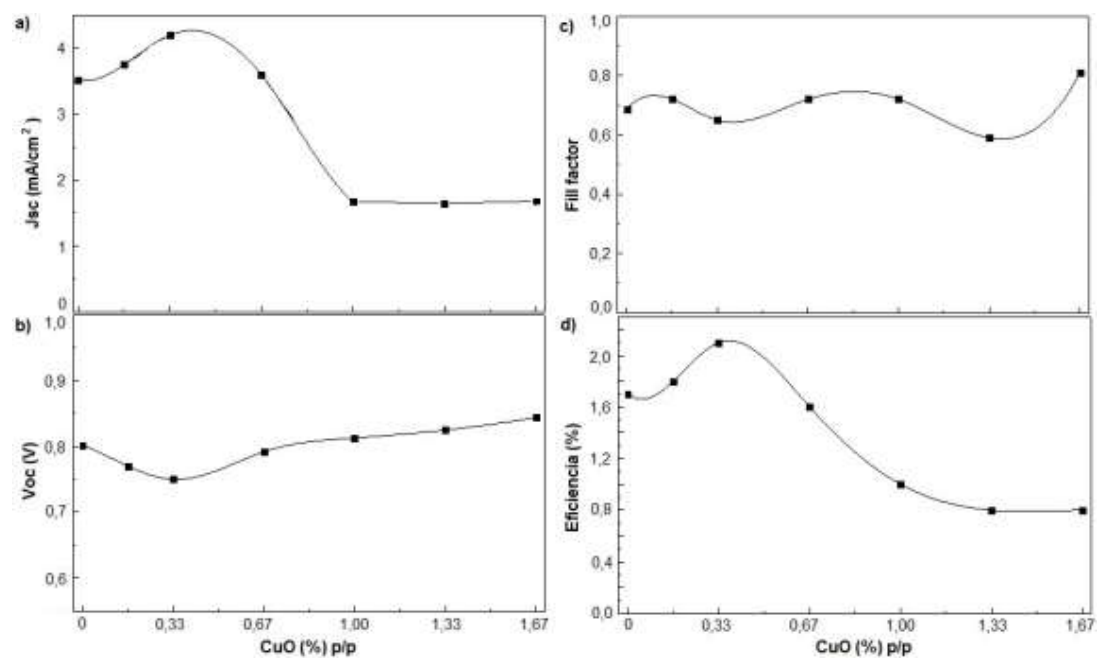

Figura 12. Curvas de a) Densidad de corriente de corto circuito (Jsc), b) voltaje de circuito abierto (Voc), c) Fill factor (FF) y d) eficiencia solar de la celda ( $\eta$ ) vs. cantidad de cobre presente en el electrodo de trabajo.

La figura 13 presenta la curva I-V para la celda fabricada con capa de bloqueo (de 10 recorridos) y un recubrimiento $\mathrm{TiO}_{2}-\mathrm{CuO}(0,33 \% \mathrm{p} / \mathrm{p})$, que resulta ser la celda óptima.

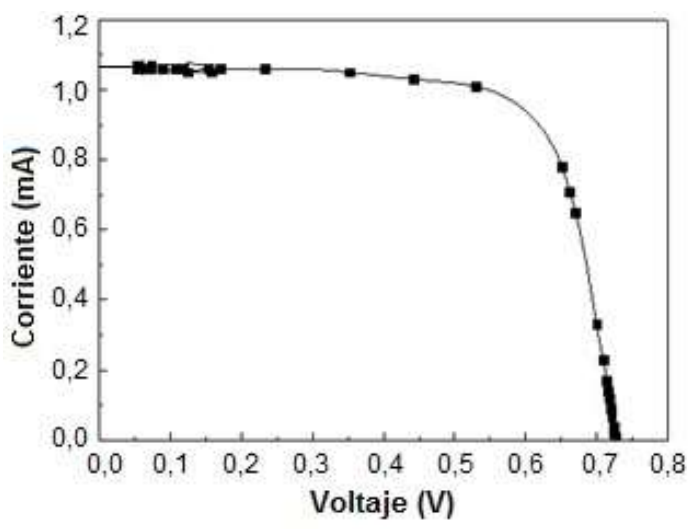

Figura 13. Curva característica ( $\mathrm{I}$ - V) de la celda solar de $\mathrm{TiO}_{2}-\mathrm{CuO}$ modificada con $0,33 \%$ de $\mathrm{CuO}$ y fabricada con capa de bloqueo.

Tabla 3. Valores de la celda óptima.

\begin{tabular}{cccc}
\hline Jsc $\left(\mathbf{m A} / \mathbf{c m}^{\mathbf{2}}\right)$ & $\operatorname{Voc}(\mathbf{V})$ & $\mathbf{F F}(\%)$ & $\boldsymbol{\eta}(\boldsymbol{\%})$ \\
\hline 4,20 & 0,73 & 0,69 & 2,1 \\
\hline
\end{tabular}




\section{Eficiencia en la conversión fotón incidente a electrón generado (IPCE)}

El sistema empleado para estas mediciones estuvo constituido por una lámpara de xenón de $1000 \mathrm{~W}$, un sistema óptico constituido por un monocromador, un semiespejo, un espectrofotómetro y un sistema de adquisición de datos. Se trabajó con un arreglo de 2 electrodos, considerando el rango de longitudes de onda entre 400 y $700 \mathrm{~nm}$.

La película de $\mathrm{TiO}_{2}-\mathrm{CuO}(0,33 \%$ p/p de $\mathrm{CuO}$ con la película de bloqueo de 10 recorridos $)$ presentó un máximo de $56,9 \%$ para una longitud de onda de $530 \mathrm{~nm}$, que corresponde al máximo de absorción de colorante13. Mientras que la película de solo $\mathrm{TiO}_{2}$ presentó un máximo de 52,1\%, para la misma longitud de onda. Por otro lado, la película de $\mathrm{TiO}_{2}-\mathrm{CuO}$ $(1,67 \%$ p/p de $\mathrm{CuO})$, mostró un máximo de $22,6 \%$ en $548 \mathrm{~nm}$.

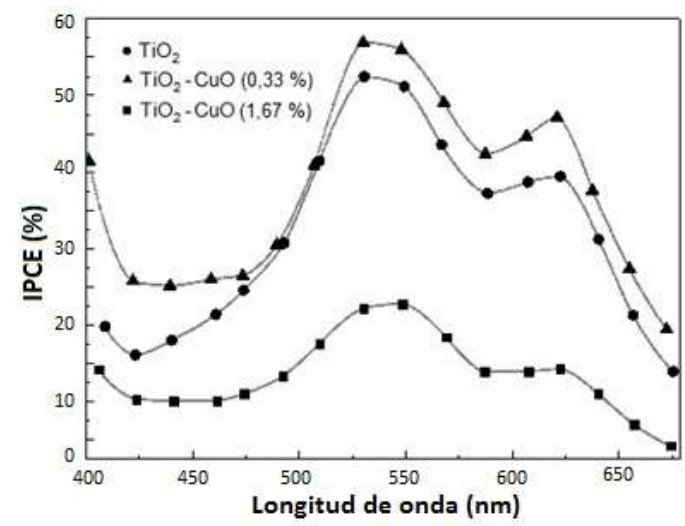

Figura 14. Eficiencia de conversión de fotón incidente a electrón generado.

\section{Voltametría cíclica}

Se realizó la voltametría cíclica en un sistema de tres electrodos, donde el electrodo de trabajo fue un recubrimiento de $\mathrm{TiO}_{2}-\mathrm{CuO}$, preparado con diferentes concentraciones de $\mathrm{CuO}(0,0,33$ y $1,67 \%)$ depositado sobre un sustrato conductor. Las medidas se realizaron en oscuridad (líneas sólidas) y bajo iluminación (líneas punteadas). Para la iluminación se empleó una lámpara UV, con componente en el rango UV-A y UV-B. El potencial de barrido fue de $50 \mathrm{mV} / \mathrm{s}$ para todas las medidas. El electrodo de referencia fue $\mathrm{Ag} / \mathrm{AgCl}$ saturado en sal de cloruro de potasio, el contraelectrodo fue una punta de platino y se midió en un electrolito de ferricianuro de potasio. Se usó el potenciostato / galvanostato, modelo 263A Princeton Applied Research.

\section{Electrodo de trabajo mesoporoso de $\mathrm{TiO}_{2}$ con $0 \%$ de $\mathrm{CuO}$}

En la figura 15 se muestran los voltagramas cíclicos de una película de $\mathrm{TiO}_{2}$. En oscuridad (línea negra) se observó un pico catódico alrededor de $-0,12 \mathrm{~V}$ correspondiente a la reducción del $\mathrm{Fe}^{+2} / \mathrm{Fe}^{+3}$, y el pico anódico alrededor de $0,29 \mathrm{~V}$ del $\mathrm{Fe}^{+2} / \mathrm{Fe}^{+3}$. En el análisis bajo iluminación (líneas punteadas) se observa en los voltagramas que la corriente aumentó 
respecto al proceso en oscuridad, indicando que la iluminación (predominante a $350 \mathrm{~nm}$ ) sobre el $\mathrm{TiO}_{2}$ favorece las reacciones redox del sistema $\mathrm{Fe}^{+3} / \mathrm{Fe}^{+2}$.

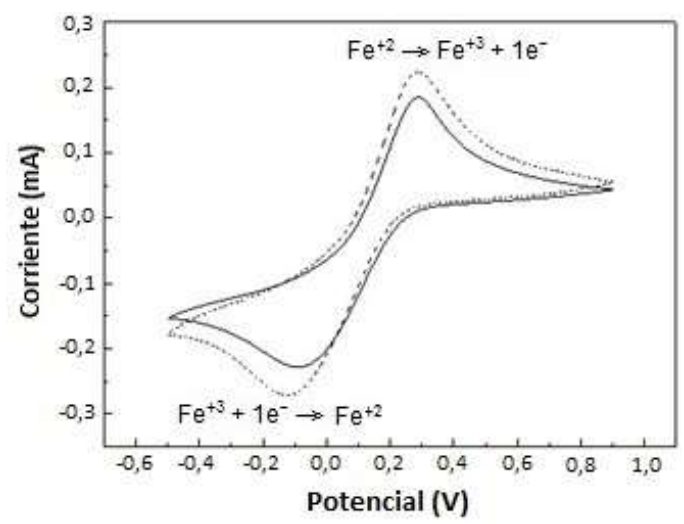

Figura 15. Voltagramas cíclicos de la película de $\mathrm{TiO}_{2}$, proceso sin luz (línea sólida) y proceso con luz (línea punteada).

\section{Electrodo de trabajo mesoporoso modificado $\mathrm{TiO}_{2}-\mathrm{CuO}$ con 0,33 y $1,67 \%$ de $\mathrm{CuO}$}

La figura 16 a) corresponde a los voltagramas cíclicos de una película de $\mathrm{TiO}_{2}-\mathrm{CuO}$ con $0,33 \%$ de $\mathrm{CuO}$, durante la medición sin luz, además de los picos de oxidación/reducción del electrolito, se observan también dos picos pequeños adicionales en el recorrido anódico $(0,64 \mathrm{~V})$ y en el recorrido catódico $(0,56 \mathrm{~V})$. Los cuales se atribuyen a los procesos $\mathrm{Cu}^{+1} /$ $\mathrm{Cu}^{+2} \mathrm{y} \mathrm{Cu}^{+2} / \mathrm{Cu}^{+1}$, respectivamente ${ }^{14}$. Durante el barrido bajo iluminación (líneas punteadas) se distingue un aumento de transferencia de carga en los recorridos anódico y catódico; sin embargo, en el primero, este es más notorio debido a un solapamiento de los dos picos que se observan en el proceso sin luz. Además, se observa que ambos picos se han desplazado hacia un potencial más positivo, para el caso del pico anódico, y un potencial más negativo, para el pico catódico, lo que indicaría que hay un desplazamiento de la banda de conducción del $\mathrm{TiO}_{2}$ Luego del barrido bajo iluminación, ya no se observa el pico del $\mathrm{Cu}$, lo que señalaría que el $\mathrm{Cu}$ ya no se encuentra en el electrodo.

Análogamente, en la figura 16 b) se muestran los voltagramas de la película de $\mathrm{TiO}_{2}-\mathrm{CuO}$ con $1,67 \%$ de $\mathrm{CuO}$. En el proceso sin luz se observa la presencia notoria del pico anódico $(640 \mathrm{mV})$, atribuido al proceso de oxidación de $\mathrm{Cu}^{+1} / \mathrm{Cu}^{+2}$, y catódico $(620 \mathrm{mV})$ asignado a la reducción de $\mathrm{Cu}^{+2} / \mathrm{Cu}^{+1}$. Estos picos son mucho más pronunciados que en el caso anterior donde el electrodo de trabajo contuvo $0,33 \%$ de $\mathrm{CuO}$, lo que concordaría con la mayor presencia de $\mathrm{CuO}$. Durante la voltametría con luz (líneas punteadas), los picos asignados a la oxidación/reducción del $\mathrm{Cu}^{+1} / \mathrm{Cu}^{+2}$, han desaparecido casi totalmente, lo que indica que el $\mathrm{CuO}$ ya no se encuentra más en el electrodo, obteniéndose en definitiva una reacción irreversible. 

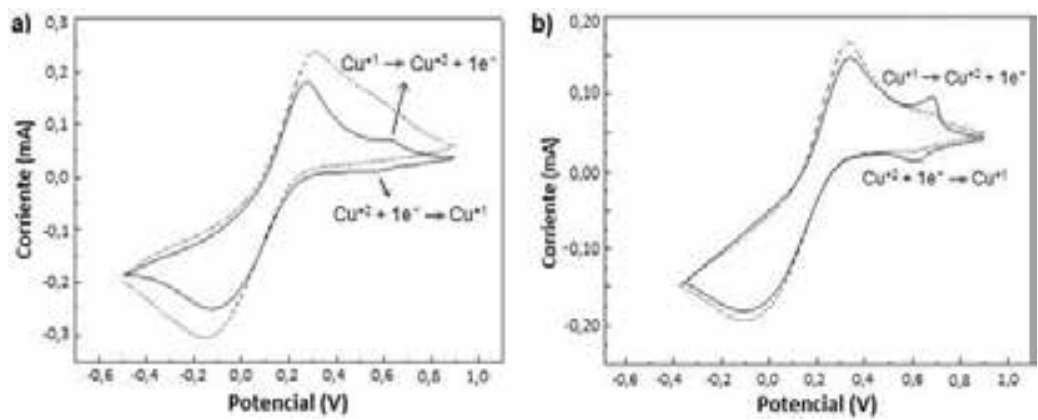

Figura 16. Voltagramas cíclicos de recubrimientos $\mathrm{TiO}_{2}-\mathrm{CuO}$ con a) $0,33 \%$ y b) $1,67 \%$ de $\mathrm{CuO}$, en procesos sin luz (línea sólida) y con luz (línea punteada)

\section{CONCLUSIONES}

Se prepararon celdas solares sensibilizadas selladas empleando electrodos de trabajo modificados $\left(\mathrm{TiO}_{2}-\mathrm{CuO}\right)$. Estos recubrimientos fueron depositados sobre sustratos conductores pre-cubiertos con una capa de bloqueo de $\mathrm{TiO}_{2}$. Las condiciones óptimas para la concentración del $\mathrm{CuO}$ y la capa de bloqueo se determinaron considerando el valor de la eficiencia solar que alcanzó $2,1 \%$ para una concentración de $0,33 \mathrm{de} \mathrm{CuO}$, y una capa crecida con 10 recorridos de rociado.

La caracterización de las partículas de $\mathrm{CuO}$ mostró que estas se presentaron en forma de conglomerados cristalinos de tamaños entre 50 y $500 \mathrm{~nm}$ constituidos a su vez por dominios cristalinos de aproximadamente $10 \mathrm{~nm}$. Los cuales no pudieron ser identificados en los electrodos de trabajo modificados $\mathrm{TiO}_{2}-\mathrm{CuO}$, empleando técnicas como microscopía electrónica de barrido y FTIR, debido a la baja concentración del $\mathrm{CuO}$ en el $\mathrm{TiO}_{2}$. Sin embargo, mediante voltametría cíclica se identificaron los picos de oxidación/reducción de las especies $\mathrm{Cu}^{+1} / \mathrm{Cu}^{+2}$.

El aumento de eficiencia en las celdas solares, debido a la presencia de pequeñas cantidades de $\mathrm{CuO}(0,33 \%)$ en el recubrimiento mesoporoso de $\mathrm{TiO}_{2}$, disminuiría reacciones de recombinación. Sin embargo, el aumento posterior de $\mathrm{CuO}$ en los electrodos modificados ocasionó una disminución de la corriente en las celdas solares, lo que podría estar asociado a aspectos como un pobre anclaje del colorante sobre la superficie del $\mathrm{CuO}^{15}$, o al desplazamiento de los niveles de energía del semiconductor con relación al colorante y el electrolito, lo que no favorecería la inyección de los electrones que atraviesan el electrodo nanoestructurado para llegar al contacto posterior.

\section{AGRADECIMIENTOS}

El presente trabajo fue desarrollado bajo el auspicio del Consejo Nacional de Ciencia, Tecnología e Innovación Tecnológica (CONCYTEC). 
Los autores agradecen a la Dra. Elizabeth Carrera y al Dr. Luis Angelats, por los análisis de SEM, al Bach. Carlos Castillo por las mediciones de IPCE, y al Bach. Alejandro Arana, por su ayuda en las mediciones de voltametría cíclica. Uno de nosotros (M.M.G.) agradece al Instituto General de Investigación de la Universidad Nacional de Ingeniería por la financiación otorgada como Docente Investigador.

\section{REFERENCIAS BIBLIOGRÁFICAS}

1. Boyle, G. Renewable Energy. 2004, University Press: Oxford.

2. O'Regan; B. and M. Grätzel. A low-cost, high-efficiency solar cell based on dyesensitized colloidal TiO2 films. Nature, 1991. 353: 737-740.

3. Nazeeruddin, M.K., E. Baranoff, and M. Grätzel. Dye-sensitized solar cells: A brief overview. Sol. Energ., 2011. 85(6): 1172-1178.

4. Gratzel, M. Recent advances in sensitized mesoscopic solar cells. Acc Chem Res, 2009. 42(11): 1788-98.

5. Galicia, M., J. Solís, and M. Gómez. Fabricación y caracterización de celdas solares sensibilizadas de TiO2 modificado con nanopartículas de NiO. Rev. Soc. Quím. Perú, 2016. 81(1): 87-99

6. Nazario, R., et al. Evaluación de capas de bloqueo en celdas solares sensibilizadas de óxido de titanio nanoestructurado. Rev. Soc. Quím. Perú, 2015. 81(2): 109-122.

7. Jiang, C., et al. Influences of alcoholic solvents on spray pyrolysis deposition of $\mathrm{TiO} 2$ blocking layer films for solid-state dye-sensitized solar cells. J. Solid State Chem., 2013. 198: $197-202$.

8. J.L. Capelo-Martínez. "Ultrasound in Chemistry: Analytical Applications", Editorial Wiley-VCH, Weinhein, Alemania. 2009, 1.

9. J.C. Williams. "Treatise on Materials Science and Technology", Editorial Elsevier, New York, USA. 1976, 173

10. TOPAS Academic V5, http://www.topas-academic.net/2012 (último acceso 08 noviembre 2016)

11. Gharagozlou, M. and S. Naghibi. Preparation of vitamin B12-TiO2 nanohybrid studied by TEM, FTIR and optical analysis techniques. Mater. Sci. Semicond. Process., 2015. 35: 166-173.

12. Bhattacharjee, A. and M. Ahmaruzzaman. Facile synthesis of 2-dimensional $\mathrm{CuO}$ nanoleaves and their degradation behavior for Eosin Y. Mater. Lett., 2015. 161: 20-25.

13. Saxena, V., et al. Co-sensitization of N719 and RhCL dyes on carboxylic acid treated $\mathrm{TiO}_{2}$ for enhancement of light harvesting and reduced recombination. Org. Electron, 2013. 14(11): 3098-3108.

14. Shakeela, K., et al. Electrochemical behaviour of $\mathrm{Cu}(\mathrm{II}) / \mathrm{Cu}(\mathrm{I})$ redox couple in 1-hexyl3-methylimidazolium chloride ionic liquid. J. Chem. Sci., 2015. 127(1): 133-140.

15. Park, J.-Y., et al. Copper and nitrogen doping on $\mathrm{TiO} 2$ photoelectrodes and their functions in dye-sensitized solar cells. J. Power Sources, 2016. 306: 764-771. 